\title{
A Case Report
}

\section{Mosaic Down syndrome with Chronic Myeloid Leukemia: A case report}

\author{
Md Habib Khan', Md Golam Rabbani², Sanjoy Kumar Chakraborty ${ }^{3}$, Rajat Sanker Roy Biswas ${ }^{4}$
}

\begin{abstract}
A female of fifty (50) with low IQ and normal physical configuration consulted with a general physician with features of infection. After initial clinical and laboratory evaluation she was found having hematological malignancy, chronic myeloid leukemia(CML) and was given antimitotic drugs. As she was not responded well bone marrow study was done and Philadelphia(Ph) chromosome was also searched for by karyotyping. Incidentally, she showed mosaic variety of Down's syndrome without any Ph chromosome. Then her treatment plan was changed and she responded well with changed schedule. Down syndrome is an important risk factor for hematological malignancy. Mosaic variety of Down patient (incidence is 1\%) has little physical abnormality other than low IQ as in this case.
\end{abstract}

Key words: CML-Chronic myeloid leukemia, Down syndrome, Mosaicism, Ph chromosome-Philadelphia chromosome.

\section{Introduction:}

Chronic myeloid leukemia (CML) was probably the first form of leukemia to be recognized as a distinct entity. In 1845, two patients were described as having massive splenomegaly associated with leukocytosis, ${ }^{1}$ which seemed to be a novel entity not explained by the other causes of splenomegaly, such as tuberculosis, that were already widely accepted in the 1840s. The first important clue to its pathogenesis came only very much later, when in 1960 newly developed techniques for studying human cells in mitosis allowed Nowell and Hungerford to detect a consistent chromosomal abnormality ${ }^{2}$ later termed the Philadelphia $(\mathrm{Ph})$ chromosome and identified as $22 q-$, in persons with this disease. In 1973, Rowley observed that the Ph chromosome resulted from a reciprocal

1. Associate Professor and Head, Department of Anatomy, BGC Trust Medical College hospital, Chittagong.

2. Associate Professor and Head, Department of Haematology, Chittagong Medical College hospital, Chittagong.

3. Assistant Professor, Department of Anatomy, BGC Trust Medical College, Chittagong.

4. MD - Thesis Part student, Internal, Medicine, Chittagong Medical College.

Correspondence: *Dr. Md. Habib Khan translocation that also involved chromosome 9; the abnormality is now designated $t(9 ; 22)$ $(q 34 ; q 11){ }^{3}$ In the 1980 s, the Ph chromosome was shown to carry a unique fusion gene, termed $B C R$ $A B L,{ }^{4}$ the generation of which is now believed to be the principal cause of the chronic phase of CML.

Down syndrome, or Down's syndrome, trisomy 21, or trisomy $\mathrm{G}$, is a chromosomal disorder caused by the presence of all or part of an extra 21st chromosome. It is named after John Langdon Down, the British physician who described the syndrome in 1866. The disorder was identified as a chromosome 21 trisomy by Jérôme Lejeune in 1959.

Individuals with Down syndrome tend to have a lowerthan-average cognitive ability, often ranging from mild to moderate disabilities. A small number have severe to profound mental disability. The average IQ of children with Down syndrome is around 50 , compared to normal children with an IQ of $100 .^{5}$ The incidence of Down syndrome is estimated at 1 per 800 to 1,000 births, although it is statistically much more common with older mothers. Other factors may also play a role. 


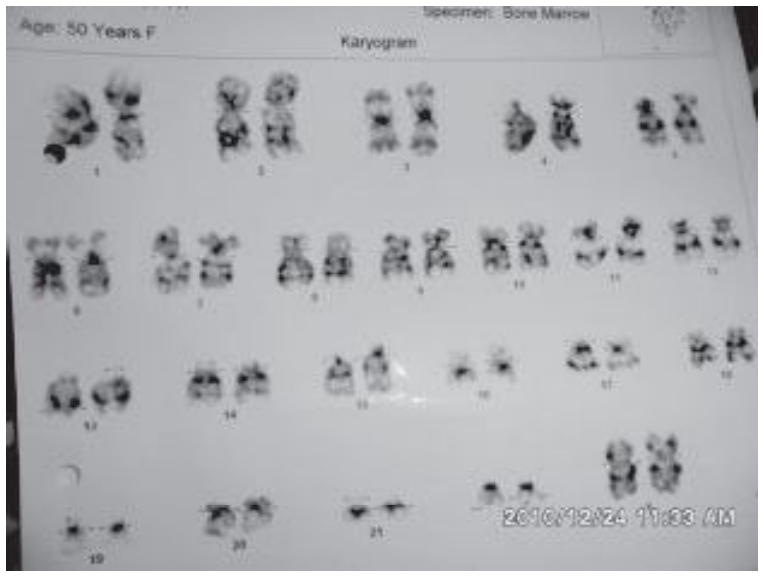

Fig.-1: Abnormal Karyotype with extra Chromosome of 21

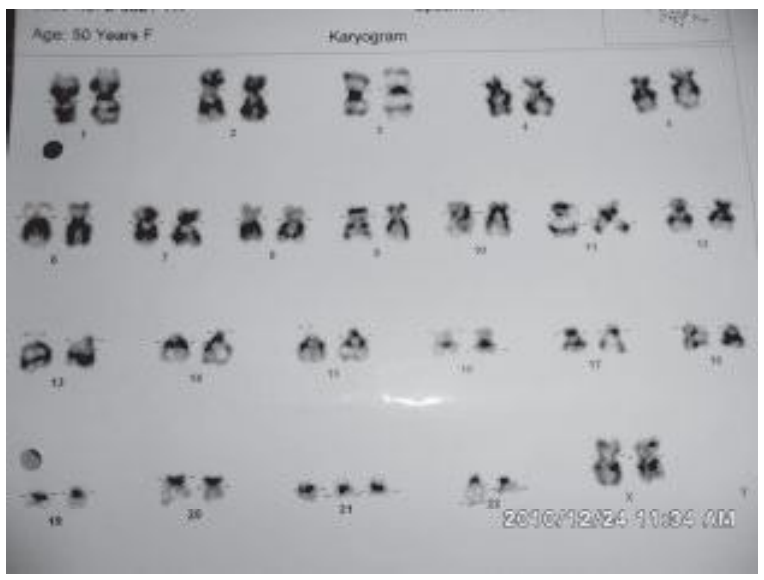

Fig.-2: Normal karyotype of cells of the same patient

\section{Case presentation:}

A 50 years old nondiabetic, normotensive asian female, illiterate with low IQ, living in a rural area of Bangladesh presented to a general physician for fever, weakness, palpitation and diarrhea. She was the last child of her parents who had more 8 healthy siblings After evaluation he found the patient has some dehydration, moderate anaemia, bony tenderness and a asymptomatic huge splenomegaly (6cm from costal margin). After correction of dehydration the patient was referred to a medicine consultant in the city where on hematological analysis there were WBC- 70,000/ $\mathrm{cmm}$, Neutrophil- 42\%, Lymphocyte- 14\%, Basophil - $14 \%$, Myelocyte $-28 \%$, Platelet count- 510 thou/ $\mathrm{cmm}$ and $\mathrm{Hb}-10.8 \mathrm{gm} / \mathrm{dl}$. Peripheral Blood
Film(PBF) examination concluded a case of CML. The patient was then referred to a hematologist who gave her blood transfusion with Hydroxyurea without bone marrow examination. Initially patient's condition improved, spleen size decreased clinically but the poor patient discontinued the treatment, later and she became anemic again with symptoms. She was given again a new regime of Busalphan and Mercaptopurine for three weeks though her condition remain unaltered. Another hematologist gave her Imatinib with blood transfusion but patient's spleenic size was increasing and anemia remained persistent and myelocyte did not disappear from the PBF. Lastly the patient was advised for bone marrow study with chromosomal analysis to see the presence of Philadelphia chromosome. After the procedure the sample was sent to India for chromosomal analysis In bone marrow study the features of CML with some features of marrow fibrosis was observed but myelofibrosis could not be excluded due to unavailability of reticulin stain. From karyotype study it was seen that 18 cells showed $46, X X$ pattern I and $47, X X+21$ pattern in other 2 cells that is a mosaic variety of Down syndrome.(Figure-1 and 2 ) without any Philadelphia chromosome. She was again given Hydroxyurea and blood transfusion and Imatinib was stopped. Her condition improved after that. Myelocyte disappeared from peripheral blood and spleenic size also decreased clinically. At present pateint is on regular follow-up.

\section{Discussion:}

Down syndrome is a chromosomal abnormality characterized by the presence of an extra copy of genetic material on the 21st chromosome, either in whole (trisomy 21) or part (such as due to translocations). The extra chromosomal material can come about in several distinct ways. A typical human karyotype is designated as $46, \mathrm{XX}$ or $46, \mathrm{XY}$, indicating 46 chromosomes with an $X X$ arrangement typical of females and 46 chromosomes with an $X Y$ arrangement typical of males. ${ }^{7}$

Types are: 1. Trisomy 2. Mosaicism 3. Robertsonian translocation 4 Duplication of a portion of chromosome 21

Mosaicism can be defined as the presence in an individual or in a tissue of two or more cell lines 
which differ in their genetic constitution but are derived from a single zygote i.e. they have the same genetic origin. Chromosome mosaicism usually results from non- disjunction occurring in an early embryonic mitotic division with the persistence of more than one cell line. Mosaicism accounts for 1$2 \%$ of all clinically recognized cases of Down's syndrome ${ }^{9}$

The options for treating patients with newly diagnosed CML have changed fundamentally since the introduction of imatinib. Until recently, interferon alfa was generally accepted as the best single agent for treating CML in the chronic phase in patients who were not eligible for allogeneic stem-cell transplantation. The drug may cause a wide range of side effects, especially in older persons, but it induces complete or nearly complete cytogenetic responses in 10 to 30 percent of patients and probably prolongs survival to a greater extent than hydroxyurea. ${ }^{8}$,

It is now generally accepted that allogeneic hematopoietic stem-cell transplantation can cure CML in selected patients, but stem-cell transplantation is still associated with an appreciable risk of complications and death, due principally to graft versus host disease and opportunistic infections. The decision about whether to offer transplantation to a given patient is aided to some degree by knowledge that the factors that influence the risk of transplantation-related death are now reasonably well defined and include the patient's age, the phase of disease, the duration of disease, the degree of donor-recipient histocompatibility, and the donor's sex. Thus, for example, a young patient with newly diagnosed CML in the chronic phase who has an HLA-identical sibling donor can expect to fare much better after transplantation than an older patient with accelerated-phase disease who has a less well matched, unrelated donor ${ }^{8}$.

\section{Conclusion:}

The patient is a female with a case of Down syndrome, mosaic variety confirmed by chromosomal analysis suffering from CML which is also evidenced by bone marrow study. Initially she responded to Hydroxyurea as after initiation of therapy her splenic size reduced and PBF showed few myelocyte but switching over to Imatinib has not helped her. Her karyotype has no $\mathrm{Ph}$ chromosome and it is the more acceptable explanation of non response to Imatinib. Now after getting the karyogram with negative $\mathrm{Ph}$ chromosome again she was given Hydroxyurea and she showed satisfactory response in PBF. She is getting regular follow-up with acceptable heath till date.

Ethical Conflict: Permission has been taken from the patient regarding using her health information in academic use as well as reporting it in print media.

\section{References:}

1. Geary CG. The story of chronic myeloid leukaemia. Br J Haematol 2000;110:2-11

2. Nowell PC, Hungerford DA. A minute chromosome in human chronic granulocytic leukemia. Science 1960;132:1497.

3 Rowley JD. A new consistent chromosomal abnormality in chronic myelogenous leukaemia identified by quinacrine fluorescence and Giemsa staining. Nature 1973;243:290-3.

4. Shtivelman E, Lifshitz B, Gale RP, Canaani E. Fused transcript of abl and bcr genes in chronic myelogenous leukaemia. Nature 1985; 315: 550-4.

5. Liptak, Gregory S. (December 2008). "Down Syndrome (Trisomy 21; Trisomy G)" Merck Manual. http://www Merck .com/mmhe/ sec23/ ch266/ch266b. html\# sec23-ch266 ch266b-420 Retrieved 2010-07-30. "Symptoms"

6. Ikeda K, Goto S. Additional anomalies in Hirschsprung's disease: an analysis based on the nationwide survey in Japan. Z Kinderchir. 1986 Oct;41(5):279-81

7. Chronic Myeloid Leukemia Trialists' Collaborative Group. Interferon alfa versus chemotherapy for chronic myeloid leukemia: a meta-analysis of seven randomized trials. $\mathrm{J}$ Natl Cancer Inst 1997;89:1616-20.

8. Druker BJ, Talpaz M, Resta DJ, et al. Efficacy and safety of a specific inhibitor of the BCR$A B L$ tyrosine kinase in chronic myeloid leukemia. N Engl J Med 2001;344:1031-1037

9. Robert FM, Ivan BY. Emerys Elements of Medical Genetics. $11^{\text {th }}$ Edition. Churchill Livingstone, Milan, p-52 